\title{
Fabrication of GaN-Based White Light-Emitting Diodes on Yttrium Aluminum Garnet-Polydimethylsiloxane Flexible Substrates
}

\author{
Lung-Chien Chen, Wen-Wei Lin, and Jun-Wei Chen \\ Department of Electro-Optical Engineering, National University of Technology, 1, Sec. 3, Chung-Hsiao E. Road, Taipei 106, Taiwan
}

Correspondence should be addressed to Lung-Chien Chen; ocean@ntut.edu.tw

Received 15 April 2015; Accepted 31 August 2015

Academic Editor: Charles C. Sorrell

Copyright (C) 2015 Lung-Chien Chen et al. This is an open access article distributed under the Creative Commons Attribution License, which permits unrestricted use, distribution, and reproduction in any medium, provided the original work is properly cited.

This study concerns the characteristics of white GaN-based light-emitting diode (LED) on flexible substrates. The thin film GaNbased blue LEDs were directly transferred from sapphire onto the flexible polydimethylsiloxane (PDMS) substrates by laser lift-off (LLO) process. The PDMS substrates were incorporated 10-40\% cerium doped yttrium aluminum garnet phosphor, YAG:Ce ${ }^{3+}$, and formed the GaN-based white LEDs. The white LEDs prepared by the GaN-based LEDs on the YAG-PDMS substrates reveal one peak at $470 \mathrm{~nm}$ corresponding to the emission of the GaN-based LED and a broadband included five weak peaks caused by YAG:Ce ${ }^{3+}$ phosphors.

\section{Introduction}

LEDs are regarded as the most important light source in next-generation solid-state lighting to advantages in energy efficiency, long life, high reliability, and multiple applications $[1,2]$. In recent years, due to the more benefits with flexible substrate, the fabrication of lighting sources on flexible substrates has been studied vigorously to develop, such as biomedical applications, deformable display, wearable devices, and flexible LEDs [3-6].

Several approaches have presented flexible lightemitting diodes (F-LEDs) by transferring microstructured $\mathrm{GaAs} / \mathrm{GaN}$ to flexible substrates [7-10]. And the majority methods have produced flexible and highly efficient thin film GaN-based LEDs by laser lift-off (LLO) technology [11-15].

In this study, we have prepared a polydimethylsiloxane (PDMS) as a substrate which had the advantages of being flexible, low cost, good chemicophysical properties and being good optically transparent. The fabrication of thin film GaN-based light-emitting diodes (GaN-based LEDs) was transferred from sapphire to flexible substrate by LLO method and produced the white light-emitting diode device.

\section{Experimental}

2.1. PDMS Substrate Production Process. PDMS solution was produced by mixing silicone resin agent (A agent) and curing agent ( $\mathrm{B}$ agent) into liquid in weight ratio for $10: 1$. Then, the yellow phosphor was incorporated into the PDMS solution and stirred uniformly. Afterward, the PDMS incorporated YAG phosphor solution was placed in a vacuum chamber for $30 \mathrm{~min}$ so that bubbles disappeared. Polytetrafluoroethene (PTFE) is sprayed on the sapphire substrate to avoid PDMS sticking on sapphire. Then, PDMS was uniformly coated on a sapphire substrate by spin coater (speed of $300 \mathrm{rpm}$ and a coating time of $40 \mathrm{sec}$ ). Subsequently, the PDMS substrate was treated at temperature of $120^{\circ} \mathrm{C}$ for $30 \mathrm{~min}$ to produce PDMS substrate. Finally, the cured PDMS substrate incorporated YAG phosphor (YAG-PDMS substrate) was removed from the sapphire substrate to produce white LED.

2.2. Preparation of White LED. The GaN-based lightemitting diode comprised a $3 \mu \mathrm{m}$ thick GaN:Si layer, five pairs of undoped InGaN/GaN multiple-quantum-wells (InGaNGaN MQWs), and a $0.5 \mu \mathrm{m}$ thick layer of GaN:Mg sequentially on a (0001) oriented patterned sapphire substrate with a GaN buffer layer that was grown by metal-organic chemical 


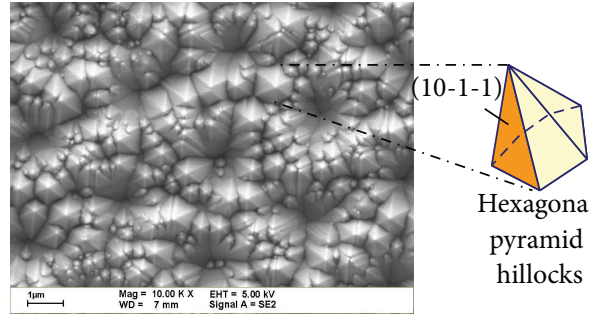

(a)

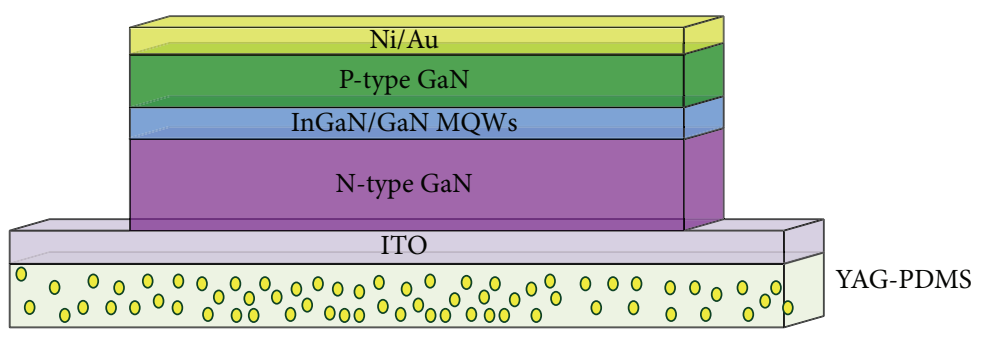

(b)

Figure 1: (a) Surface of the n-GaN epitaxial layer after KOH solution etching and (b) GaN-based LED after LLO process was mounted on flexible YAG-PDMS substrate.

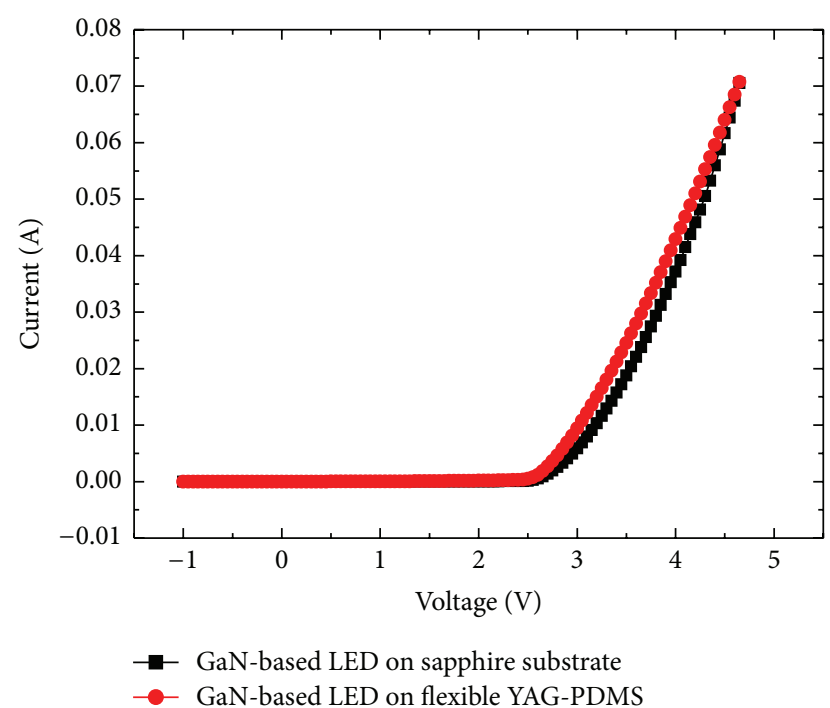

FIGURE 2: $I$ - $V$ curves of LEDs on sapphire substrate and YAG-PDMS substrate, respectively.

vapor deposition (MOCVD). Ni/Au (45 nm/15 nm thickness) layers are deposited on the $\mathrm{p}-\mathrm{GaN}$ as ohmic contact, respectively. Next, p-GaN/InGaN-GaN MQWs/n-GaN structure is separated from the sapphire substrate by LLO method using $248 \mathrm{~nm} \mathrm{KrF}$ laser after bonding to glass temporary substrate. The wafer was etching using $\mathrm{KOH}$ solution for $30 \mathrm{~min}$ to remove residue gallium oxide. The surface of the $n-G a N$ epitaxial layer was observed hexagonal pyramid hillocks, as shown in Figure 1(a). Then, the structure was transferred onto the YAG-PDMS substrate with ITO contact layer to complete the GaN-based white LEDs. Figure 1(b) demonstrates the structure of the GaN-based LEDs on the YAG-PDMS substrate.

\section{Results and Discussion}

Figure 2 plots $I-V$ curves of LED on sapphire substrate and YAG-PDMS substrate, respectively. I- $V$ characteristics of the GaN-based LED on the YAG-PDMS substrate exhibited less turn-on voltage and resistivity than that of the GaNbased LED on the sapphire substrate. The comparably good electrical characteristics of the GaN-based LED on flexible PDMS substrates that proposed the laser lift-off technology

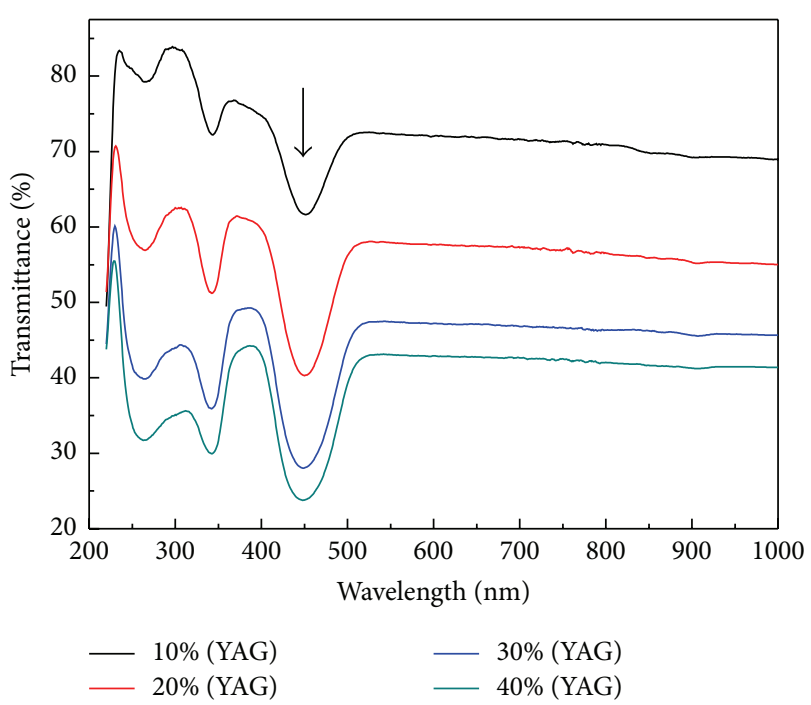

FIGURE 3: Transmittance spectra of YAG-PDMS substrates with various YAG incorporation amount.

would not degrade device electrical performance and have better trend owing to the vertical operating device. However, leakage was observed at low forward bias. That means the stress between the GaN epitaxial layer and the flexible YAGPDMS substrate due to removing the sapphire substrate.

Figure 3 shows the transmittance spectra of YAG-PDMS substrates with various YAG incorporation amount in weight percentage. The transmittance decreases when the incorporation amount in PDMS increases owing to the absorption by the YAG phosphors. There is a most deep absorption valley at around $450 \mathrm{~nm}$ in all samples, assigned to the $4 \mathrm{f}\left({ }^{2} \mathrm{~F}_{5 / 2}\right) \rightarrow$ $5 \mathrm{~d}_{2}$ transitions of $\mathrm{Ce}^{3+}$ ions, as shown in Figure 3. Therefore, the YAG-PDMS is suitable for the exciting source of the GaN LED with emitting wavelength of about $450 \mathrm{~nm}$.

Figure 4 shows the normalized electroluminescence (EL) spectra of the LEDs on YAG-PDMS with different incorporated concentration at the RT. According to Figure 4, the RT EL spectra of the GaN LEDs on the YAG-PDMS substrates reveal one peak, denoted as peak $A$, that is, at $470 \mathrm{~nm}$, and a broadband included five weak peaks, denoted as peaks $I_{1}, I_{2}, I_{3}, I_{4}$, and $I_{5}$ at $2.56 \mathrm{eV}(484 \mathrm{~nm}), 2.36 \mathrm{eV}(525 \mathrm{~nm})$, $2.27 \mathrm{eV}(547 \mathrm{~nm}), 2.15 \mathrm{eV}(578 \mathrm{~nm})$, and $2.02 \mathrm{eV}(615 \mathrm{~nm})$, respectively. The intensity of the broadband increases when 


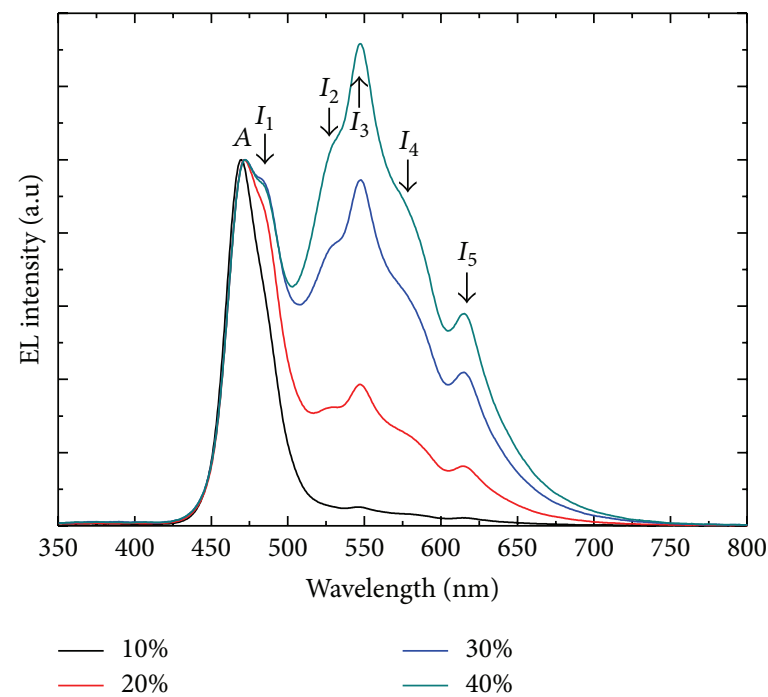

FIGURE 4: EL spectra obtained with different incorporation amount.

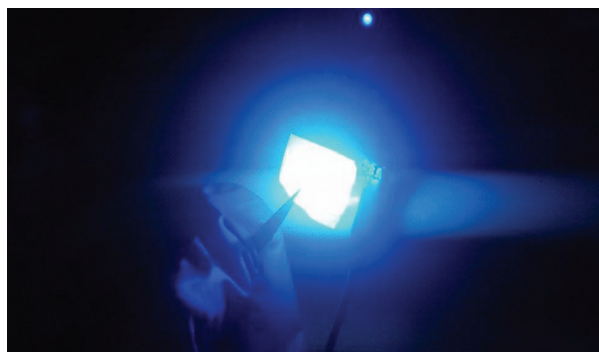

(a)

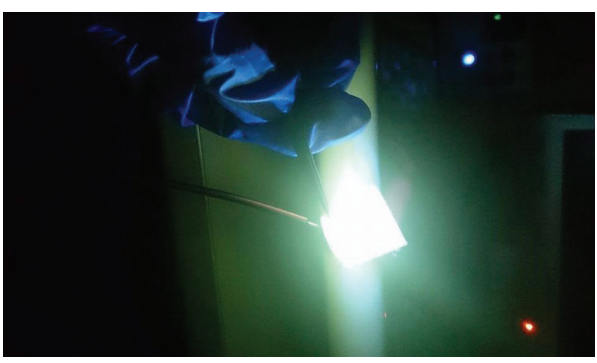

(c)

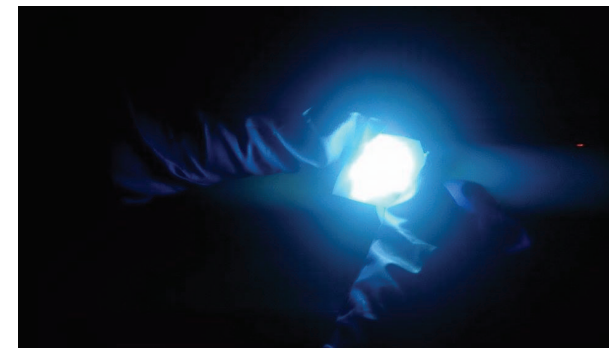

(b)

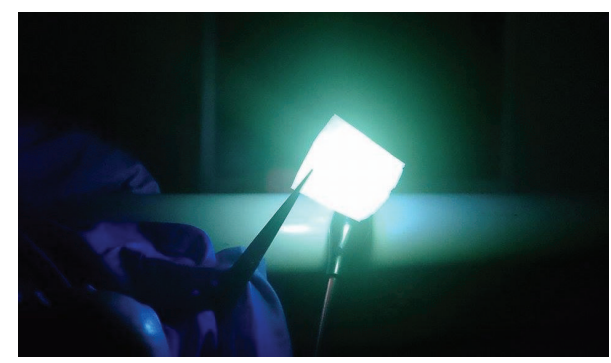

(d)

FIGURE 5: Operating photos of LED on YAG-PDMS substrates with various incorporation amounts: (a) 10\%, (b) 20\%, (c) 30\%, and (d) 40\% concentration of phosphor.

the incorporated concentration increases from $10 \%$ to $40 \%$. Peak $A$ is attributed to the GaN blue LED with $470 \mathrm{~nm}$ emitting wavelength as exciting light source. The broadband that included five weak peaks may be corresponding to ${ }^{5} \mathrm{D}_{4} \rightarrow{ }^{7} \mathrm{~F}_{6},{ }^{5} \mathrm{D}_{1} \rightarrow{ }^{2} \mathrm{~F}_{5},{ }^{5} \mathrm{D}_{4} \rightarrow{ }^{7} \mathrm{~F}_{5},{ }^{5} \mathrm{D}_{4} \rightarrow{ }^{7} \mathrm{~F}_{4}$, and ${ }^{5} \mathrm{D}_{4} \rightarrow{ }^{7} \mathrm{~F}_{3}$ level transitions of $\mathrm{Ce}^{3+}$ ions in the YAG phosphors, respectively [16-18].

The color of EL is nearly white, as shown in Figure 5. The color is from white-bluish to white-yellowish when the incorporation amount of YAG phosphors in PDMS substrates increases from $10 \%$ to $40 \%$. The white light may have contributed to the wide emission band ranging from 400 to $750 \mathrm{~nm}$. The chromaticity coordinates of the YAG phosphors at 10, 20, 30, and 40\% in PDMS substrates are presented in the CIE (Commission Internationale De L'éclairage) chromaticity diagram, as shown in Figure 6. With the increasing incorporated concentration of YAG, the chromaticity coordinates move in white light area from $x=$ $0.3666, y=0.3446$ (correlated color temperature $(\mathrm{CCT})=$ $6700 \mathrm{~K})$ for the sample at $10 \%$ to $x=0.3280, y=0.3762$ $(\mathrm{CCT}=5666 \mathrm{~K})$ for the sample at $40 \%$. The variation of CCT is only $\sim 15 \%$ when the incorporated concentration of YAG increases from $10 \%$ to $40 \%$. Therefore, the YAG phosphors in PDMS substrate are suitable for flexible solid-state lighting because it has a stable white light color when the incorporated concentration is in the range of $10-40 \mathrm{wt} \%$. 


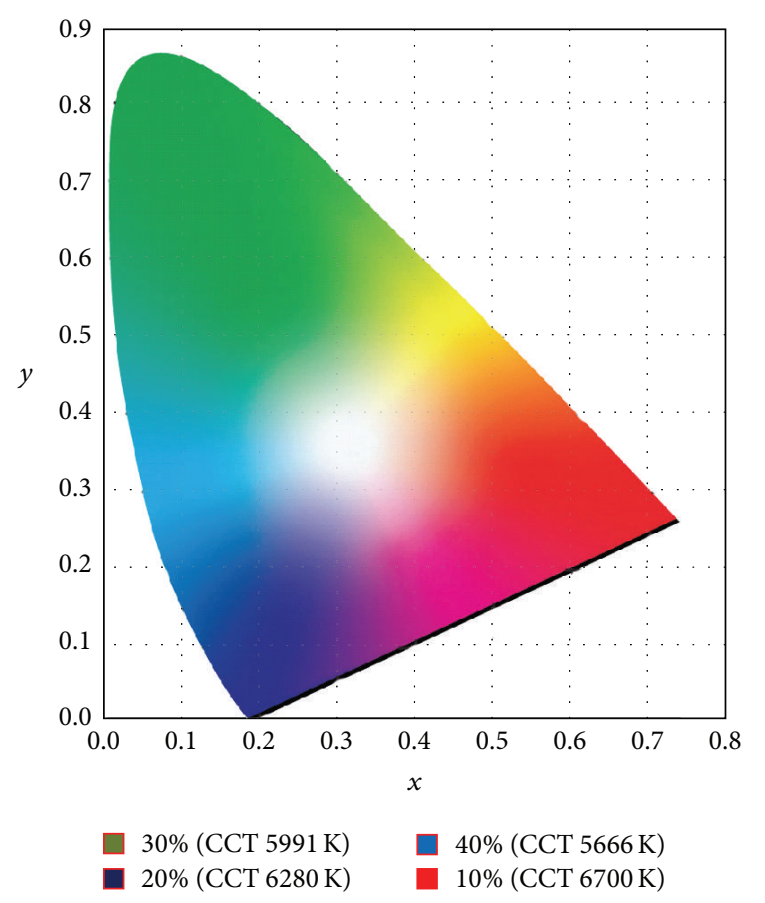

FIGURE 6: Chromaticity coordinates of the GaN-based LEDs on the YAG-PDMS substrates.

\section{Conclusions}

In summary, characteristics of white GaN-based LEDs on YAG-PDMS substrates are reported. The white light may have contributed to the wide emission band ranging from 400 to $750 \mathrm{~nm}$. The chromaticity coordinates of the YAG phosphors at $10,20,30$, and $10 \%$ in YAG-PDMS substrates are presented in the CIE chromaticity diagram. With the increasing incorporated concentration of YAG, the chromaticity coordinates move in white light area from $x=0.3666, y=0.3446$ (CCT $=$ $6700 \mathrm{~K})$ for the sample at $10 \%$ to $x=0.3280, y=0.3762$ $(\mathrm{CCT}=5666 \mathrm{~K})$ for the sample at $40 \%$. Therefore, the YAG phosphors in PDMS substrate are suitable for flexible solidstate lighting because it has a stable white light color when the incorporated concentration is in the range of $10-40 \mathrm{wt} \%$.

\section{Conflict of Interests}

The authors declare that there is no conflict of interests regarding the publication of this paper.

\section{Acknowledgment}

Financial support of this paper was provided by the Ministry of Science and Technology, China, under Contract no. NSC 103-2221-E-027-029-MY2.

\section{References}

[1] R. H. Horng, C. C. Chiang, H. Y. Hsiao, X. Zheng, D. S. Wuu, and H. I. Lin, "Improved thermal management of GaN/sapphire light-emitting diodes embedded in reflective heat spreaders," Applied Physics Letters, vol. 93, no. 11, Article ID 111907, 2008.
[2] R.-H. Horng, H.-Y. Hsiao, C.-C. Chiang, D.-S. Wuu, Y.-L. Tsai, and H.-I. Lin, "Novel device design for high-power ingan/sapphire leds using copper heat spreader with reflector," IEEE Journal on Selected Topics in Quantum Electronics, vol. 15, no. 4, pp. 1281-1286, 2009.

[3] R.-H. Kim, D.-H. Kim, J. Xiao et al., "Waterproof AlInGaP optoelectronics on stretchable substrates with applications in biomedicine and robotics," Nature Materials, vol. 9, no. 11, pp. 929-937, 2010.

[4] S.-I. Park, Y. Xiong, R.-H. Kim et al., "Printed assemblies of inorganic light-emitting diodes for deformable and semitransparent displays," Science, vol. 325, no. 5943, pp. 977-981, 2009.

[5] M. Koo, S. Y. Park, and K. J. Lee, "Biointegrated flexible inorganic light emitting diodes," Nanobiosensors in Disease Diagnosis, vol. 1, pp. 5-15, 2012.

[6] M. S. White, M. Kaltenbrunner, E. D. Głowacki et al., "Ultrathin, highly flexible and stretchable PLEDs," Nature Photonics, vol. 7, no. 10, pp. 811-816, 2013.

[7] H.-S. Kim, E. Brueckner, J. Song et al., "Unusual strategies for using indium gallium nitride grown on silicon (111) for solidstate lighting," Proceedings of the National Academy of Sciences of the United States of America, vol. 108, no. 25, pp. 10072-10077, 2011.

[8] S.-I. Park, A.-P. Le, J. Wu, Y. Huang, X. Li, and J. A. Rogers, "Light emission characteristics and mechanics of foldable inorganic light-emitting diodes," Advanced Materials, vol. 22, no. 28, pp. 3062-3066, 2010.

[9] T.-I. Kim, Y. H. Jung, J. Song et al., "High-efficiency, microscale GaN light-emitting diodes and their thermal properties on unusual substrates," Small, vol. 8, no. 11, pp. 1643-1649, 2012.

[10] R.-H. Jiang, C.-F. Lin, Y.-C. Huang et al., "Separating In GaN membranes from GaN/sapphire templates through a crystallographic-etch-limited process," RSC Advances, vol. 3, no. 32, pp. 13446-13450, 2013.

[11] S. J. Kim, "Effect of residual stress of thin and thick layers on laser lifted-off light emitting diodes," Journal of the Electrochemical Society, vol. 158, no. 9, pp. 904-907, 2011.

[12] H.-Y. Kuo, S.-J. Wang, P.-R. Wang et al., "Use of elastic conductive adhesive as the bonding agent for the fabrication of vertical structure GaN-based LEDs on flexible metal substrate," IEEE Photonics Technology Letters, vol. 20, no. 7, pp. 523-525, 2008.

[13] C.-H. Lee, Y.-J. Kim, Y. J. Hong et al., "Flexible inorganic nanostructure light-emitting diodes fabricated on graphene films," Advanced Materials, vol. 23, no. 40, pp. 4614-4619, 2011.

[14] J. Chun, Y. Hwang, Y.-S. Choi et al., "Transfer of GaN LEDs from sapphire to flexible substrates by laser lift-off and contact printing," IEEE Photonics Technology Letters, vol. 24, no. 23, pp. 2115-2118, 2012.

[15] L.-C. Chen, W.-W. Lin, and C.-A. Chiou, "Relaxation of stress in gan-based light-emitting diodes on stainless steel substrate by plasma etching using a mask of silver nanoparticles," Science of Advanced Materials, vol. 6, no. 6, pp. 1179-1183, 2014.

[16] Y. Zhang, L. Li, X. Zhang, and Q. Xi, “Temperature effects on photoluminescence of YAG: $\mathrm{Ce}^{3+}$ phosphor and performance in white light-emitting diodes," Journal of Rare Earths, vol. 26, no. 3, pp. 446-449, 2008.

[17] C.-H. Hsu, L.-C. Chen, and J.-R. Wu, "Prepared and characteristics of $\mathrm{ZnO}: \mathrm{YAG} /$ silicon nanostructure diodes prepared by 
ultrasonic spraying," International Journal of Photoenergy, vol. 2014, Article ID 128235, 6 pages, 2014.

[18] R. Kasuya, A. Kawano, T. Isobe, H. Kuma, and J. Katano, "Characteristic optical properties of transparent color conversion film prepared from YAG:Ce ${ }^{3+}$ nanoparticles," Applied Physics Letters, vol. 91, no. 11, Article ID 111916, 2007. 

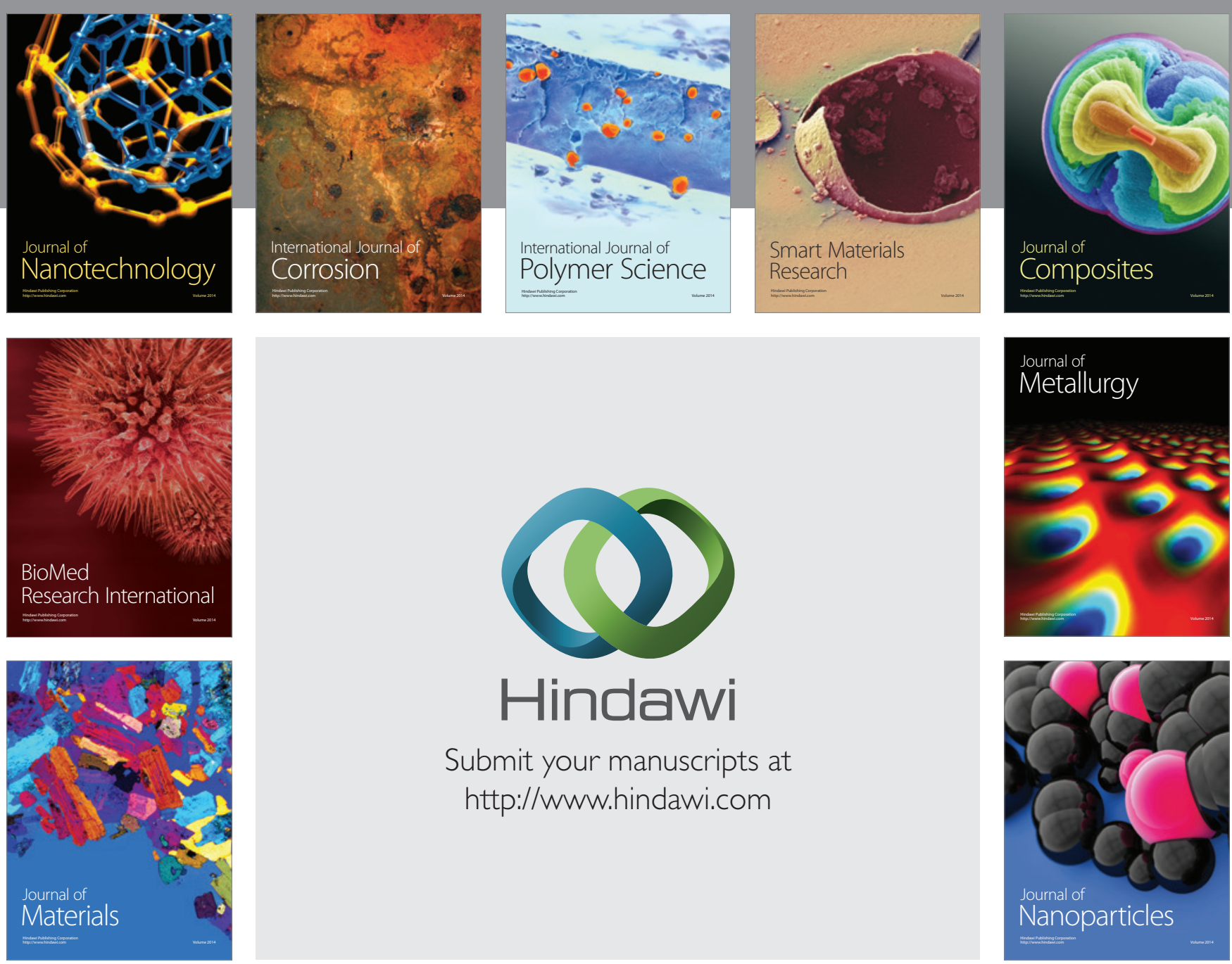

Submit your manuscripts at http://www.hindawi.com
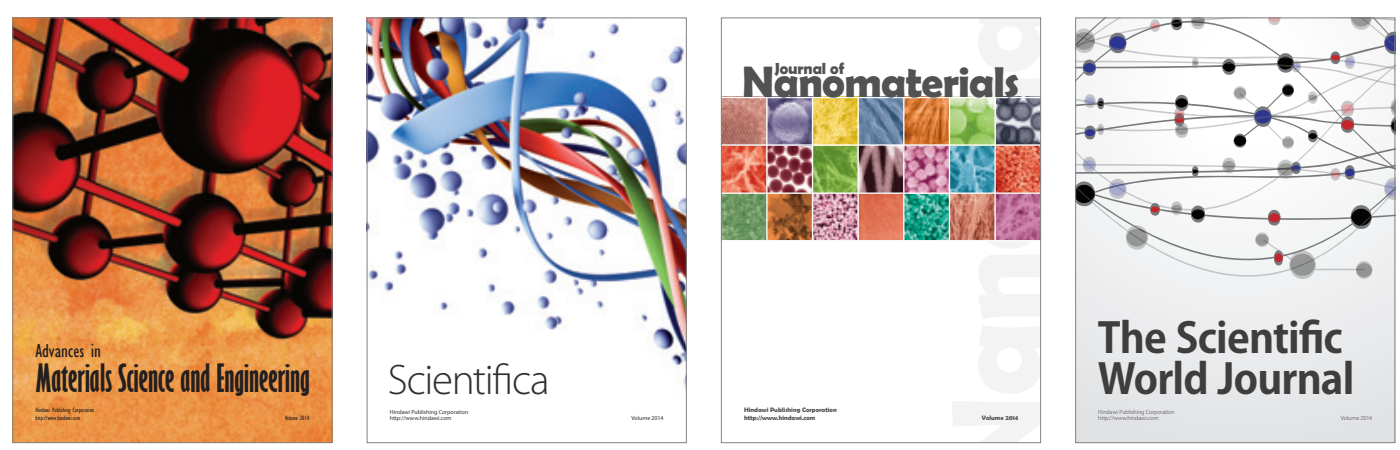

\section{The Scientific World Journal}
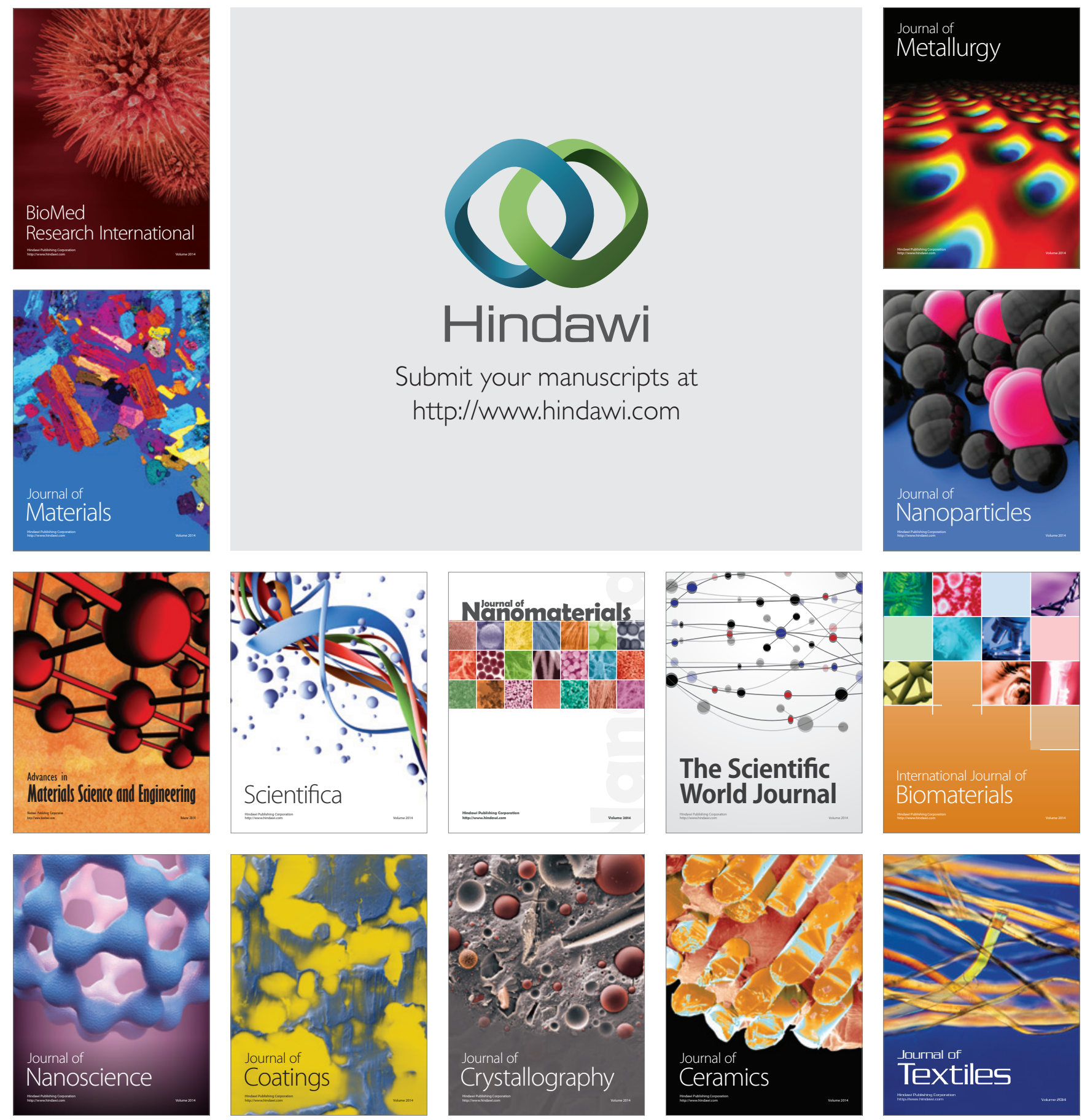